\title{
Fraud tolerance in optimal crowdfunding ${ }^{*}$
}

\author{
Matthew Ellman ${ }^{\mathrm{a}}$ and Sjaak Hurkens ${ }^{\mathrm{ab}}$
}

April 2019

\begin{abstract}
Reward-based crowdfunding enables credit-constrained entrepreneurs to raise money to develop and create innovative products. Crowdfunders' low monitoring incentives open the door to fraud. In practice, fraud is surprisingly rare. Strausz (2017) proves that crowdfunding implements the optimal ex post individually rational mechanism design outcome in an environment with entrepreneurial moral hazard and private cost information. However, ex post individual rationality precludes all crowdfunding unless fraud can be prevented with certainty. Actual crowdfunding tolerates some fraud. We show this (i) generates strictly higher profits and welfare, but (ii) cannot implement the optimal ex interim individually rational outcome.
\end{abstract}

Keywords: Crowdfunding, mechanism design, moral hazard, private information. JEL Classifications: C72, D42, D82, D86, L12, L26

\footnotetext{
${ }^{*}$ We gratefully acknowledge financial support from 2016 FBBVA grant "Innovación e Información en la Economía Digital", the Spanish Ministry via the Severo Ochoa Programme for Centres of Excellence in R\&D (SEV-2015-0563) and grants ECO2017-88129-P (AEI/FEDER, UE) (Ellman) and ECO201567171-P (MINECO) (Hurkens) and the Generalitat de Catalunya via the grant 2017-SGR-1136.

${ }^{a}$ Institute for Economic Analysis (CSIC) and Barcelona GSE. Campus UAB, 08193 Bellaterra, Spain.

${ }^{\mathrm{b}}$ Corresponding author. Emails: matthew.ellman@iae.csic.es and sjaak.hurkens@iae.csic.es.
} 


\section{Introduction}

In reward-based crowdfunding, an entrepreneur presents a project to create a new product to a crowd of potential buyers who can then pre-order it at some price $p$ over a limited period. The entrepreneur promises to produce and deliver the product if the sum of money pledged $P$ exceeds some pre-specified target $T$. If the target is not met, there is no production, pre-orders are cancelled and consumers are reimbursed. Ellman and Hurkens (2015) use mechanism design to derive the optimal $(T, p)$ strategy. They prove that crowdfunding's main benefit lies in learning about demand before sinking production costs $I$ and raising funds is not fundamental. In practice, however, crowdfunding platforms collect the money from pre-ordering buyers and transfer all the funds to the entrepreneur if the target is met, returning them if not. This creates an opportunity for fraud as the entrepreneur could pocket the aggregate funds pledged, forego production to save her costs and disappear without delivering the product. The entrepreneur has an incentive to do so whenever $\alpha P>P-I$, where $0 \leq \alpha \leq 1$ denotes the degree of moral hazard. ${ }^{1}$ Crowdfunding platforms warn customers about these risks. Risks are highest for entrepreneurs with high fixed costs and outright scammers with fake projects who always planned to run with any funds they can raise.

Strausz (2017) investigates whether popular crowdfunding schemes can deal optimally with such moral hazard concerns and suggests that they can. Concretely, he uses mechanism design techniques to solve the problem of maximizing profit in an environment with a credit-constrained entrepreneur who has private information about her cost and is subject to moral hazard. He derives two main features of his constrained efficient mechanism: it defers payments (as far as credit constraints permit) and limits the entrepreneur's information about the aggregate payment. He then argues that crowdfunding indirectly implements these two features. ${ }^{2}$ Unfortunately, Strausz (2017) restricts attention to ex post individually rational $\left(\mathrm{IR}^{x p}\right)$ mechanisms. We argue in Section 3 that this restriction is untenable in a context with fraud risk. We relax Strausz's optimization problem by disregarding $\operatorname{IR}^{x p}$. Then we characterize the relevant constrained optimum. We highlight its interesting added feature of cross-subsidization via cost-contingent transfers.

Crowdfunding's transparent pricing precludes efficient cross-subsidies: funders would reject optimal pricing when the cost is high. Nonetheless, crowdfunding can generate higher profits and welfare than Strausz's optimal IR ${ }^{x p}$ mechanism because crowdfunders tolerate the risk of high cost entrepreneurs effectively extracting a subsidy via fraud, provided low cost entrepreneurs cross-subsidize via a low price. We begin with a simple example of this fraud-tolerance point.

\footnotetext{
${ }^{1}$ The share $(1-\alpha)$ is lost; it represents the cost of running off with the money.

${ }^{2}$ Strausz (2017) does recognize in footnote 6 that actual crowdfunding cannot jointly implement both features unless the constrained optimum is efficient.
} 


\section{An example where optimal crowdfunding has fraud}

Strausz (2017) concludes that crowdfunding is completely useless if there is any probability, no matter how small, of an entrepreneur who cannot produce the good or whose cost exceeds the maximal demand. If such an entrepreneur did raise funds from consumers paying their maximal valuation or less, then she would certainly commit fraud, absconding with the funds instead of producing and delivering the product. Because cost information is private, crowdfunding can only fully prevent such fraud by not funding any project. Imposing $\operatorname{IR}^{x p}$ therefore precludes all funding. The optimal solution has no funding and no production. However, intuition and evidence strongly suggest that crowdfunders should tolerate some fraud risk if sufficiently small - as in the classic refrain, "nothing ventured, nothing gained." The following example vindicates this intuition.

Example 1. There is one consumer who privately observes his valuation: $v \in\{0,1\}$ has probability $\pi(v)$. There is one credit-constrained entrepreneur who learns her cost type privately: with probability $\rho_{1}=3 / 4$, her cost is $I_{1}=0.3$ and with probability $\rho_{2}=1 / 4$, her cost is $I_{2}>2 / 3$. The degree of moral hazard is $\alpha=0.5$. Note that $\alpha I_{2}>1-I_{2}$ so that the high cost entrepreneur prefers to run with any funds $P \leq 1$. To satisfy the $v=1$ consumer's IR ${ }^{x p}$, no money must ever be given to either type of entrepreneur: any crowdfunding schedule $(T, p)$ inducing production by the low cost entrepreneur would also attract the high cost entrepreneur who would abscond. So the optimal IR ${ }^{x p}$ mechanism has no production and zero surplus for entrepreneur and consumer. However, this no-trade outcome characterized by Strausz (2017) is strictly Pareto dominated by the outcome from crowdfunding schedule $(T, p)$ with $T=p=0.7$, offered by both types of entrepreneur:

When the consumer pledges $p$, the high cost entrepreneur runs with the money (gaining $\alpha p>p-I_{2}$ ) whereas the low cost entrepreneur produces (gaining $p-I_{1}>\alpha p$ ). Since $\rho_{1}-p>0$, the consumer with valuation 1 is willing to pledge $p$, despite not receiving the reward when the entrepreneur has high cost.

\section{On the ex post IR constraint}

In his definition of a constrained efficient mechanism, Strausz (2017) not only imposes budget- and development-feasibility, incentive compatibility and obedience constraints but also imposes inequality constraint (29): each consumer receives at least "his outside option conditional on his own type and the project's cost structure" (p.1449, our italics). In the case of perfect information about the project's cost structure, this is just interim individual rationality, but it goes far beyond that when there is imperfect information about costs. Indeed, for the case of just one consumer, which we characterize fully in the 
next section, this assumption is identical to ex post individual rationality. ${ }^{3}$

Mechanism design problems that impose $\operatorname{IR}^{x p}$ are usually motivated by an option for the involved agents to opt out (veto or withdraw) after learning the outcome. See, for example, Forges (1999), Compte and Jehiel (2007, 2009) and Krähmer and Strausz (2015). However, the ex post constraint is unjustified and fundamentally inappropriate in the context of crowdfunding with private cost information. ${ }^{4}$ Entrepreneurs with overly high costs will pool on the crowdfunding price and threshold proposals of entrepreneurs that are attractive to consumers. Consumers may eventually learn about the entrepreneur's cost after a successful campaign, as when low cost entrepreneurs produce while high cost entrepreneurs run with the money. But they have no option to withdraw their pledge at that stage: it is then simply too late to withdraw and avoid the loss. $\operatorname{IR}^{x p}$ would be justified if crowdfunding platforms guaranteed to prosecute fraudulent entrepreneurs and fully reimburse funders, but this is clearly not the case. E.g., Kickstarter explicitly warns that "... a project may not work out the way everyone hopes. Kickstarter creators have a remarkable track record, but nothing's guaranteed. Keep this in mind when you back a project." Moreover, if such guarantees existed and were perfect, the whole moral hazard problem would disappear as no entrepreneur would ever try to run with the money.

\section{Optimal mechanism design without $\operatorname{IR}^{x p}$}

Under IR ${ }^{x p}$, there is no loss in restricting attention to mechanisms that prevent all fraud; the approach taken in Strausz (2017). Example 1 already proves that crowdfunding that tolerates some fraud generates better outcomes than this $\mathrm{IR}^{x p}$ solution achieves, but perhaps a more general mechanism can do even better? We now solve for the optimum without imposing $\operatorname{IR}^{x p}$. In particular, we provide a complete characterization of optimal mechanisms in the simplest case of one consumer and two types of entrepreneur. This uncovers a third feature, on top of transfer deferral and hiding information from entrepreneurs, of the constrained optimal mechanism: it involves restricting consumer information about the entrepreneur's cost. The intuition is straightforward. Hiding this information enables efficient cross-subsidization: e.g., consumers are then willing to pay a higher price in high cost states, raising the deferred payments where moral hazard is high.

\footnotetext{
${ }^{3}$ Ex post individual rationality only differs from (29) in also conditioning on other bidders' types. This distinction obviously disappears with a single bidder. It is also irrelevant with multiple bidders in this crowdfunding context because a bidder is only affected by other bidders' types via their impact on whether or not production is recommended and all bidders get zero when production is not recommended.

${ }^{4}$ Dominant strategy implementation would require $\operatorname{IR}^{x p}$ (as not bidding guarantees a zero payoff) but the fraud opportunity then immediately precludes production in any putative strategy-proof equilibrium.
} 


\subsection{Notation}

The set of consumers is $\mathcal{N}=\{1, \ldots, n\}$. The vector of privately observed valuations is $v=$ $\left(v_{1}, \ldots, v_{n}\right) \in\{0,1\}^{n}, \pi(v)$ denotes its probability and $n(v) \equiv \sum_{i \in \mathcal{N}} v_{i}$ denotes the number of high value consumers. An allocation $a=(t, x)=\left(t_{1}^{a}, \ldots, t_{n}^{a}, t_{1}^{p}, \ldots, t_{n}^{p}, x_{0}, x_{1}, \ldots, x_{n}\right)$ consists of ex ante and ex post transfers $t_{i}^{a}$ and $t_{i}^{p}$ from consumer $i$, the probability $x_{0} \in\{0,1\}$ that investment takes place and the probability $x_{i} \in\{0,1\}$ that consumer $i$ consumes one unit of the good. The transfers are contingent on the behavior of the entrepreneur as follows: both are paid in full when the entrepreneur produces but when the entrepreneur fraudulently runs with the ex ante transfers $t^{a}$, the entrepreneur only enjoys fraction $\alpha$ of them, wasting $(1-\alpha) t^{a}$, and ex post transfers $t^{p}$ are not paid; $\alpha>0$ captures the degree of moral hazard. A project is characterized by fixed cost $I \in \mathcal{K}$. The entrepreneur privately observes her type $k$ defining her cost $I_{k}$; type $k$ has probability $\rho_{k}>0$. A deterministic direct mechanism $(t, x)$ assigns an allocation $(t(I, v), x(I, v))$ to each demand and cost state. Consumer utility $U_{i}$ and entrepreneurial profit $\Pi$ are: ${ }^{5}$

$$
\begin{aligned}
U_{i}\left(a \mid v_{i}\right) & =x_{0}\left[v_{i} x_{i}-t_{i}^{a}-t_{i}^{p}\right]+\left(1-x_{0}\right)\left[-t_{i}^{a}\right] \\
\Pi(a \mid I) & =x_{0}\left[\sum_{i \in \mathcal{N}}\left[t_{i}^{a}+t_{i}^{p}\right]-I\right]+\left(1-x_{0}\right)\left[\alpha \sum_{i \in \mathcal{N}} t_{i}^{a}\right]
\end{aligned}
$$

\subsection{Constrained optimum in the simplest case}

We characterize the constrained optimum of the general mechanism in the case where $n=1$ and the entrepreneur has two possible costs. The interesting case is that with $I_{1}<1 /(1+\alpha)<I_{2}{ }^{6}$ There are two subcases of interest: (a) $I_{2}>1$ and (b) $I_{2}<1$. Notice that, conditional on the consumer's valuation being 1 , it is efficient for both types of entrepreneur to produce in case (b) and for only type 1 to produce in case (a).

We maximize expected social surplus subject to IR and IC constraints. Production is infeasible when the single consumer has valuation 0 , so we need only consider the case where the consumer has valuation 1 for the good. This event has probability $\pi(1)$.

A stochastic direct mechanism must decide for each entrepreneurial type, $k=1,2$, the probability $\gamma_{k}$ to instruct her to produce (that is, $x_{0}\left(I_{k}, 1\right)=1$ ) and the contractual transfers $t_{k}^{a}, t_{k}^{p}$ in that event (she then receives total price $p_{k}=t_{k}^{a}+t_{k}^{p}$ if obedient), and the probability $\gamma_{k}^{\prime}=1-\gamma_{k}$ to instruct her to not produce (that is, $x_{0}\left(I_{k}, 1\right)=0$ ) and her corresponding unconditional payment $p_{k}^{\prime}{ }^{7}$ To relax the moral hazard constraint when

\footnotetext{
${ }^{5}$ These expressions correct Strausz's (2017) equations (6) and (7) to deal with $x_{0} \neq 1$. Strausz (2017) tacitly restricted to contingent transfers, but we extend to allow allocations with unconditional transfers.

${ }^{6} \mathrm{It}$ is straightforward to see that the first-best can be implemented if $I_{1}<I_{2}<1 /(1+\alpha)$, while no production can be implemented if $1 /(1+\alpha)<I_{1}<I_{2}$.

${ }^{7}$ There is no need for an unconditional transfer with $x_{0}=1$ nor incentive transfers $t^{a}, t^{p}$ with $x_{0}=0$. Also, the good is consumed whenever produced, i.e. $x_{1}=x_{0}$.
} 
recommending production, an optimal mechanism sets ex ante transfer $t_{k}^{a}=I_{k}$, deferring $t_{k}^{p}=p_{k}-I_{k}$; recall that if the entrepreneur does not produce, she only enjoys $\alpha t_{k}^{a}$ and $t_{k}^{p}$ is not paid. the entrepreneur is simply paid $p_{k}^{\prime}$.

The general optimization problem is thus

$$
\max _{\gamma_{1}, \gamma_{1}^{\prime}, \gamma_{2}, \gamma_{2}^{\prime}, p_{1}, p_{1}^{\prime}, p_{2}, p_{2}^{\prime}} \pi(1)\left[\gamma_{1} \rho_{1}\left(1-I_{1}\right)+\gamma_{2} \rho_{2}\left(1-I_{2}\right)\right]
$$

subject to

$$
\begin{aligned}
& \rho_{1}\left[\gamma_{1}\left(1-p_{1}\right)+\gamma_{1}^{\prime}\left(-p_{1}^{\prime}\right)\right]+\rho_{2}\left[\gamma_{2}\left(1-p_{2}\right)+\gamma_{2}^{\prime}\left(-p_{2}^{\prime}\right)\right] \geq 0 \\
& \gamma_{1}=0 \text { or } p_{1}-I_{1} \geq \alpha I_{1} \\
& \gamma_{2}=0 \text { or } p_{2}-I_{2} \geq \alpha I_{2} \\
& \gamma_{1}\left(p_{1}-I_{1}\right)+\gamma_{1}^{\prime} p_{1}^{\prime} \geq \gamma_{2} \max \left\{p_{2}-I_{1}, \alpha I_{2}\right\}+\gamma_{2}^{\prime} p_{2}^{\prime} \\
& \gamma_{2}\left(p_{2}-I_{2}\right)+\gamma_{2}^{\prime} p_{2}^{\prime} \geq \gamma_{1} \alpha I_{1}+\gamma_{1}^{\prime} p_{1}^{\prime} \\
& 0 \leq \gamma_{1}, 0 \leq \gamma_{1}^{\prime}=1-\gamma_{1} \\
& 0 \leq \gamma_{2}, 0 \leq \gamma_{2}^{\prime}=1-\gamma_{2} \\
& p_{1}^{\prime}, p_{2}^{\prime} \geq 0
\end{aligned}
$$

Notice that constraint $\left(\mathrm{IR}^{i}\right)$ is the interim individual rationality constraint of the consumer with valuation 1. Strausz (2017, inequality (29)) instead imposes the following which is precisely ex post individual rationality in this setting (cf., Footnote 3):

$$
\gamma_{k}\left(1-p_{k}\right)+\gamma_{k}^{\prime}\left(-p_{k}^{\prime}\right) \geq 0 \quad(\forall k=1,2)
$$

Proposition 1. Let $n=1, R=\rho_{1} / \rho_{2}>0,0<I_{1}(1+\alpha)<1<I_{2}(1+\alpha)$. Define

$$
R_{1} \equiv \frac{\alpha I_{1}}{1-(1+\alpha) I_{1}} \text { and } R_{2} \equiv \frac{I_{1}\left[(1+\alpha) I_{2}-1\right]}{I_{1}^{2}+I_{2}-(2+\alpha) I_{1} I_{2}}
$$

(a) When $I_{2}>1$, the first-best has $\left(\gamma_{1}, \gamma_{2}\right)=(1,0)$ and:

If $R \geq R_{1}$, this can be implemented by setting $p_{2}^{\prime}=R\left(1-p_{1}\right) \geq \alpha I_{1}$ and any $p_{1} \in$ $\left[\frac{R+I_{1}}{R+1}, 1-\frac{\alpha I_{1}}{R}\right]$. Raising $p_{1}$ transfers rent from high to low cost entrepreneurs.

If $R<R_{1}$, no production can be implemented: $\gamma_{k}=p_{k}=p_{k}^{\prime}=0 \forall k=1,2$.

(b) When $I_{2}<1$, the first-best has $\left(\gamma_{1}, \gamma_{2}\right)=(1,1)$; it cannot be implemented. Instead: If $R \geq R_{2}$, the constrained optimum is uniquely determined by $\left(\gamma_{1}, \gamma_{2}\right)=\left(1, \gamma_{2}\right)$ where $\gamma_{2} \in(0,1)$ is defined by $p_{2}^{\prime}=0$ and binding constraints $\left(I R^{i}\right),\left(I C_{12}\right)$ and $\left(M H_{2}\right)$. If $R_{1} \leq R<R_{2}$, the constrained optimum is uniquely determined by $\left(\gamma_{1}, \gamma_{2}\right)=\left(1, \gamma_{2}\right)$ where $\gamma_{2} \in(0,1)$ is defined by $p_{2}^{\prime}=0$ and binding constraints $\left(I R^{i}\right),\left(I C_{12}\right)$ and $\left(I C_{21}\right)$; the range $\left(R_{1} \leq R<R_{2}\right)$ is non-empty if and only if $\alpha>\left(1-I_{2}\right) / I_{1}$.

If $R<\min \left\{R_{1}, R_{2}\right\}$, no production can be implemented: $\gamma_{k}=p_{k}=p_{k}^{\prime}=0 \forall k=1,2$. 
The proof in the Appendix shows that no expected surplus is lost in having $\left(\mathrm{IR}^{i}\right)$ bind. So the focal solution of Proposition 1 also maximizes expected profit.

Observe that Example 1's parameters imply $R>R_{1}$ and $R>R_{2}$, and lie in case (a) if $I_{2}>1$. That example showed how tolerating fraud gave a feasible Pareto improvement over no production. The proposition now reveals that the optimal solution does not involve fraud, but rather a "legitimated" payment $\left(p_{2}^{\prime}\right)$ to type 2 for revealing her type; this payment avoids the social waste $(1-\alpha) I_{1}{ }^{8}$

In case (b), it is efficient to have type 2 produce with probability 1 , but the constrained optimum always has $\gamma_{2}<1$. We illustrate this possibility and an interesting aspect of the solution by reconsidering Example 1 in the specific case of $I_{2}=0.7$.

Example 2. Let $\alpha=1 / 2, I_{1}=0.3, I_{2}=0.7$ and $R=\rho_{1} / \rho_{2}=3$. In this case, $R>R_{2}$. Let $p_{1}=\frac{453}{460}, p_{2}=\frac{21}{20}, \gamma_{1}=1$ and $\gamma_{2}=\frac{21}{23}$. It is easily verified that all constraints are satisfied, with $\left(I R^{i}\right),\left(I C_{12}\right)$ and $\left(M H_{2}\right)$ binding.

Note that if there were perfect information about the entrepreneur's cost, there would be no production for type 2 because $\alpha I_{2}>1-I_{2}$. Hence, the example shows that private information may in fact alleviate incentive problems, contrary to Strausz's finding that private information always intensifies them (Strausz, 2017, p.1431, 1442).

Returning to the general picture, hiding cost information is generically valuable for increasing profits and welfare over the optimal $\mathrm{IR}^{x p}$ solution. To see this, note that consumers would never agree to pay $p_{2}>1$ or $p_{2}^{\prime}>0$ if able to learn the cost level.

\section{Concluding remarks}

We have argued why $\operatorname{IR}^{x p}$ is inadequate in the context of moral hazard and private cost information. Example 1 showed how actual reward-based crowdfunding can lead to a strict Pareto improvement over the optimal $\mathrm{IR}^{x p}$ mechanism by tolerating some fraud. So platforms should screen out enough (but not necessarily all) projects with excessive costs. We also prove that fraud-tolerant reward-based crowdfunding cannot always implement the optimal mechanism. In our characterization for the simplest case of one consumer and two types of entrepreneur, the optimum can require stochastic production and subsidies across cost states. Cross-subsidization of high cost entrepreneurs permits higher deferred payments or paying for non-production as appropriate. This is more efficient than having high cost entrepreneurs extract an effective cross-subsidy through fraud. The subtler features of our optimal mechanism may put off funders or exacerbate adverse selection, so future research should endogenize these possibilities. Also, introducing investmentbased returns may outperform pure reward-based crowdfunding.

\footnotetext{
${ }^{8}$ To avoid using $p_{2}^{\prime}$ ("paying for nothing"), the mechanism could approximate the solution by instead having type 2 produce with a very small probability $\gamma_{2}$ in return for a very high price $p_{2}=\alpha I_{1} / \gamma_{2}$.
} 


\section{Appendix: Proof of Proposition 1}

We begin with a number of observations that help simplify the optimization problem. First, note that $I_{1}<I_{2}$ implies that, if $\gamma_{2}>0, p_{2}-I_{1}>p_{2}-I_{2} \geq \alpha I_{2}$, where the weak inequality is just $\left(M H_{2}\right)$. So $\gamma_{2} \max \left\{p_{2}-I_{1}, \alpha I_{2}\right\}=\gamma_{2}\left(p_{2}-I_{1}\right)$.

Second, if $\gamma_{1}=0$, then $\gamma_{2}=0$. Suppose instead that $\gamma_{2}>0$. Then $\left(I C_{12}\right)$ and $\left(I C_{21}\right)$ imply that $p_{1}^{\prime}>p_{1}^{\prime}$, a clear contradiction. Setting all prices equal to zero as well as $\gamma_{1}=\gamma_{2}=0$, implements the no production solution with no waste. This has zero surplus, so we henceforth restrict attention to $\gamma_{1}>0$. From $\left(M H_{1}\right)$, it then follows that $p_{1}>0$.

Third, without loss of generality, we can set $p_{1}^{\prime}=0$. This is clear when $\gamma_{1}=1$. If $\left(1-\gamma_{1}\right) p_{1}^{\prime}>0$, then one can reduce $p_{1}^{\prime}$ and increase $p_{1}$ while keeping $\gamma_{1}$ and $\gamma_{1} p_{1}+(1-$ $\left.\gamma_{1}\right) p_{1}^{\prime}$ constant. This relaxes constraints $\left(M H_{1}\right)$ and $\left(I C_{12}\right)$ and affects neither the other constraints nor the objective function.

Fourth, $\gamma_{2}<1$. This obviously holds for $\gamma_{2}=0$, while if $\gamma_{2}>0, p_{2} \geq(\alpha+1) I_{2}>1$ by $\left(M H_{2}\right)$. $\left(I R^{i}\right)$ then implies that $p_{1}<1<p_{2}$. This in turn implies that $\gamma_{2}<1$ because of $\left(I C_{12}\right)$.

Fifth, without loss of generality $\left(I R^{i}\right)$ binds: an increase in $p_{1}$ relaxes $\left(I C_{12}\right)$, tightens $\left(I R^{i}\right)$ and does not affect the objective function or any other constraints.

Finally, note that $\left(M H_{1}\right)$ is implied by $\left(I C_{12}\right)$ and $\left(I C_{21}\right)$. Hence, we can ignore this constraint in the optimization problem, which can therefore be rewritten as follows:

$$
\max _{\gamma_{1}, \gamma_{2}, p_{1}, p_{2}, p_{2}^{\prime}} \gamma_{1} \rho_{1}\left(1-I_{1}\right)+\gamma_{2} \rho_{2}\left(1-I_{2}\right)
$$

subject to

$$
\begin{aligned}
& -\left[\gamma_{1} \rho_{1}\left(1-p_{1}\right)+\gamma_{2} \rho_{2}\left(1-p_{2}\right)+\left(1-\gamma_{2}\right) \rho_{2}\left(-p_{2}^{\prime}\right)\right]=0 \\
& \gamma_{2}\left[(\alpha+1) I_{2}-p_{2}\right] \leq 0 \\
& \gamma_{2}\left(p_{2}-I_{1}\right)+\left(1-\gamma_{2}\right) p_{2}^{\prime}-\gamma_{1}\left(p_{1}-I_{1}\right) \leq 0 \\
& \gamma_{1} \alpha I_{1}-\gamma_{2}\left(p_{2}-I_{2}\right)-\left(1-\gamma_{2}\right) p_{2}^{\prime} \leq 0 \\
& 0<\gamma_{1} \leq 1 \\
& 0 \leq \gamma_{2}<1 \\
& p_{2}^{\prime} \geq 0 \\
& p_{1}>0
\end{aligned}
$$

The optimal solution $\left(\gamma_{1}, \gamma_{2}, p_{1}, p_{2}, p_{2}^{\prime}\right)$ has $\gamma_{1}=1$, because otherwise $\left(\tilde{\gamma}_{1}, \tilde{\gamma}_{2}, p_{1}, p_{2}, \tilde{p}_{2}^{\prime}\right)$ would be strictly better for $\tilde{\gamma}_{k}=\gamma_{k}(1+\varepsilon)$ and $\left(1-\tilde{\gamma}_{2}\right) \tilde{p}_{2}^{\prime}=(1+\varepsilon)\left(1-\gamma_{2}\right) p_{2}^{\prime}$ for some small $\varepsilon>0$.

Using Lagrange multipliers $\lambda$ for the binding $\left(I R^{i}\right)$ constraint and $\mu_{1}, \mu_{2}, \mu_{3} \geq 0$ for 
the respective inequality constraints, we write the Lagrangian $\mathcal{L}$ as follows:

$$
\begin{aligned}
\mathcal{L}= & \rho_{1}\left(1-I_{1}\right)+\gamma_{2} \rho_{2}\left(1-I_{2}\right)+\lambda\left[\rho_{1}\left(1-p_{1}\right)+\gamma_{2} \rho_{2}\left(1-p_{2}\right)-\left(1-\gamma_{2}\right) \rho_{2} p_{2}^{\prime}\right] \\
& -\mu_{1} \gamma_{2}\left[(\alpha+1) I_{2}-p_{2}\right]-\mu_{2}\left[\gamma_{2}\left(p_{2}-I_{1}\right)+\left(1-\gamma_{2}\right) p_{2}^{\prime}-\left(p_{1}-I_{1}\right)\right] \\
& -\mu_{3}\left[\alpha I_{1}-\gamma_{2}\left(p_{2}-I_{2}\right)-\left(1-\gamma_{2}\right) p_{2}^{\prime}\right]
\end{aligned}
$$

Necessary complementary slackness conditions for an optimum are

$$
\begin{aligned}
& \gamma_{2} \geq 0 \& \frac{\partial \mathcal{L}}{\partial \gamma_{2}} \leq 0 \& \gamma_{2} \frac{\partial \mathcal{L}}{\partial \gamma_{2}}=0 \\
& \frac{\partial \mathcal{L}}{\partial p_{1}}=0 \\
& p_{2} \geq 0 \& \frac{\partial \mathcal{L}}{\partial p_{2}} \leq 0 \& p_{2} \frac{\partial \mathcal{L}}{\partial p_{2}}=0 \\
& p_{2}^{\prime} \geq 0 \& \frac{\partial \mathcal{L}}{\partial p_{2}^{\prime}} \leq 0 \& p_{2}^{\prime} \frac{\partial \mathcal{L}}{\partial p_{2}^{\prime}}=0
\end{aligned}
$$

where

$$
\begin{aligned}
\frac{\partial \mathcal{L}}{\partial \gamma_{2}}= & \rho_{2}\left(1-I_{2}\right)+\lambda \rho_{2}\left[1-p_{2}+p_{2}^{\prime}\right]-\mu_{1}\left[(\alpha+1) I_{2}-p_{2}\right] \\
& -\mu_{2}\left[p_{2}-I_{1}-p_{2}^{\prime}\right]+\mu_{3}\left[p_{2}-I_{2}-p_{2}^{\prime}\right] \\
\frac{\partial \mathcal{L}}{\partial p_{1}}= & -\lambda \rho_{1}+\mu_{2} \\
\frac{\partial \mathcal{L}}{\partial p_{2}}= & \gamma_{2}\left[-\lambda \rho_{2}+\mu_{1}-\mu_{2}+\mu_{3}\right] \\
\frac{\partial \mathcal{L}}{\partial p_{2}^{\prime}}= & \left(1-\gamma_{2}\right)\left[-\lambda \rho_{2}-\mu_{2}+\mu_{3}\right]
\end{aligned}
$$

CASE 1: We first consider the candidate solutions with $\gamma_{2}=0$. This can be implemented if and only if there exist $p_{1}, p_{2}^{\prime} \geq 0$ such that

$$
\begin{aligned}
& \rho_{1}\left(1-p_{1}\right)-\rho_{2} p_{2}^{\prime}=0 \\
& p_{2}^{\prime}-\left(p_{1}-I_{1}\right) \leq 0 \\
& \alpha I_{1}-p_{2}^{\prime} \leq 0
\end{aligned}
$$

Hence, writing $R=\rho_{1} / \rho_{2}$, this requires

$$
\alpha I_{1} \leq p_{2}^{\prime}=R\left(1-p_{1}\right) \leq p_{1}-I_{1}
$$


or, equivalently,

$$
\frac{R+I_{1}}{R+1} \leq p_{1} \leq \frac{R-\alpha I_{1}}{R}
$$

which has a solution if and only if

$$
R \geq \frac{\alpha I_{1}}{1-(\alpha+1) I_{1}}=R_{1}
$$

Of course, when the latter condition is satisfied and $I_{2} \geq 1$, this is in fact the optimal solution.

CASE 2: We now consider solutions with $\gamma_{2}>0$. Hence, (12) can be replaced by $\partial \mathcal{L} / \partial \gamma_{2}=0$. Also note that if $p_{2}^{\prime}>0$, then raising $p_{2}$ and lowering $p_{2}^{\prime}$ while keeping $\gamma_{2} p_{2}+\left(1-\gamma_{2}\right) p_{2}^{\prime}$ constant, relaxes $\left(M H_{2}\right)$ while not affecting the objective function or the other constraints. So, w.l.o.g. we may set $p_{2}^{\prime}=0$ in this case.

Note that if $\mu_{2}=0$, then also $\lambda=0$ from (17). But then also $\mu_{1}=\mu_{3}=0$ from (14) and (18), because $\mu_{1} \geq 0$ and $\mu_{3} \geq 0$. This contradicts $\partial \mathcal{L} / \partial \gamma_{2}=0$ when $I_{2} \neq 1$. Hence, $\mu_{2}>0$ and the corresponding constraint $\left(I C_{12}\right)$ must bind:

$$
\gamma_{2}\left(p_{2}-I_{1}\right)=p_{1}-I_{1}
$$

Writing as before $R=\rho_{1} / \rho_{2} \in(0, \infty)$ for the relative probability of a low cost type 1 , we can rewrite the binding $\left(I R^{i}\right)$ constraint as

$$
\gamma_{2}=\frac{R\left(1-p_{1}\right)}{p_{2}-1}
$$

This is well-defined because by $\left(M H_{2}\right), p_{2} \geq(\alpha+1) I_{2}$ and the latter strictly exceeds 1 . From (20) and (21) one can express $p_{2}$ and $\gamma_{2}$ as functions of $p_{1}$ :

$$
\begin{aligned}
p_{2}\left(p_{1}\right) & =\frac{p_{1}-I_{1}-R\left(1-p_{1}\right) I_{1}}{p_{1}-I_{1}-R\left(1-p_{1}\right)} \\
\gamma_{2}\left(p_{1}\right) & =\frac{p_{1}-I_{1}+R\left(p_{1}-1\right)}{1-I_{1}}
\end{aligned}
$$

Note that $\gamma_{2}\left(p_{1}\right)$ is a linear, strictly increasing function and that $0<\gamma_{2}\left(p_{1}\right)<1$ if and only if $p_{1} \in\left(p_{\text {min }}, 1\right)$, where $p_{\text {min }}=\frac{I_{1}+R}{1+R}$. Note that $p_{2}\left(p_{1}\right)$ is decreasing on this domain, with $\lim _{p_{1} \uparrow 1} p_{2}\left(p_{1}\right)=1$ and $p_{2}\left(p_{1}\right) \rightarrow \infty$ as $p_{1} \downarrow p_{\text {min }}$.

The question is now whether $p_{1}$ can be chosen within this domain so that constraints $\left(M H_{2}\right)$ and $\left(I C_{21}\right)$ are also satisfied. $\left(M H_{2}\right)$ requires that $p_{2}\left(p_{1}\right) \geq \hat{p}_{2}=(\alpha+1) I_{2}$. Hence, $p_{1} \leq p_{2}^{-1}\left(\hat{p}_{2}\right) \equiv \bar{p}_{1}$. It is straightforward to show that

$$
\bar{p}_{1}=\frac{\left(I_{1}+R\right)(\alpha+1) I_{2}-I_{1}(1+R)}{(1+R)(\alpha+1) I_{2}-\left(1+R I_{1}\right)}
$$


$\left(I C_{21}\right)$ requires that $Z\left(p_{1}\right) \equiv \gamma_{2}\left(p_{1}\right)\left(p_{2}\left(p_{1}\right)-I_{2}\right) \geq \alpha I_{1}$. It can be verified that $Z\left(p_{1}\right)$ is a linear function of $p_{1}$ :

$$
Z\left(p_{1}\right)=\frac{I_{1}\left(I_{2}-(R+1)\right)+I_{2} R+p_{1}\left(1+I_{1} R-I_{2}(R+1)\right)}{1-I_{1}}
$$

This function is increasing if and only if

$$
R \leq \frac{1-I_{2}}{I_{2}-I_{1}}
$$

Straightforward calculations show that

$$
Z\left(\bar{p}_{1}\right)=\frac{\left(1-I_{1}\right) I_{2} R \alpha}{I_{2}(1+R)(1+\alpha)-\left(1+I_{1} R\right)} \text { and } \lim _{p_{1} \downarrow p_{\text {min }}} Z\left(p_{1}\right)=\frac{\left(1-I_{1}\right) R}{1+R}
$$

If $I_{2}>1, Z\left(p_{1}\right)$ is strictly decreasing. Note that the objective function is now decreasing in $\gamma_{2}$ so that lower $p_{1}$ is better. There are feasible allocations if and only if $\lim _{p_{1} \downarrow p_{\text {min }}} Z\left(p_{1}\right)>\alpha I_{1}$, that is, when

$$
R>\frac{\alpha I_{1}}{1-(\alpha+1) I_{1}} \equiv R_{1}(\alpha)
$$

But in this case there exists the solution from case 1 with $\gamma_{2}=0$, which implements the first-best. If the condition is not met, there are no feasible allocation schedules with positive production.

If $I_{2}<1$, the objective function is increasing in $\gamma_{2}$. Hence, one seeks the maximal $p_{1}$. We consider two cases.

First, suppose $R \leq\left(1-I_{2}\right) /\left(I_{2}-I_{1}\right)$. Now $Z\left(p_{1}\right)$ is increasing. There is a solution with production if and only if $Z\left(\bar{p}_{1}\right) \geq \alpha I_{1}$. This is satisfied if and only if

$$
R \geq \frac{I_{1}\left((1+\alpha) I_{2}-1\right)}{I_{1}^{2}+I_{2}-(2+\alpha) I_{1} I_{2}} \equiv R_{2}(\alpha)
$$

The solution is determined by $\bar{p}_{1}$. The constraints $\left(I R^{i}\right),\left(M H_{2}\right)$ and $\left(I C_{12}\right)$ are binding.

Second, consider the case with $R>\left(1-I_{2}\right) /\left(I_{2}-I_{1}\right)$. Then $Z\left(p_{1}\right)$ is decreasing. If $\lim _{p_{1} \downarrow p_{\min }} Z\left(p_{1}\right)=\frac{\left(1-I_{1}\right) R}{1+R}>\alpha I_{1}$, there exists a unique $\tilde{p}_{1}>p_{\text {min }}$ such that $Z\left(\tilde{p}_{1}\right)=$ $\alpha I_{1}$. The optimal solution involves production by type 2 and has optimal price $p_{1}=$ $\min \left\{\tilde{p}_{1}, \bar{p}_{1}\right\}$. In particular, when $\tilde{p}_{1}<\bar{p}_{1}$, constraint $\left(M H_{2}\right)$ is slack while $\left(I C_{21}\right)$ then binds. If $\lim _{p_{1} \downarrow p_{\text {min }}} Z\left(p_{1}\right) \leq \alpha I_{1}$, there exists no implementable allocation in which type 2 produces. Note that $\lim _{p_{1} \downarrow p_{\text {min }}} Z\left(p_{1}\right) \leq \alpha I_{1}$ if and only if

$$
R \leq \frac{\alpha I_{1}}{1-(1+\alpha) I_{1}}=R_{1}(\alpha)
$$

Figure 1 illustrates. 

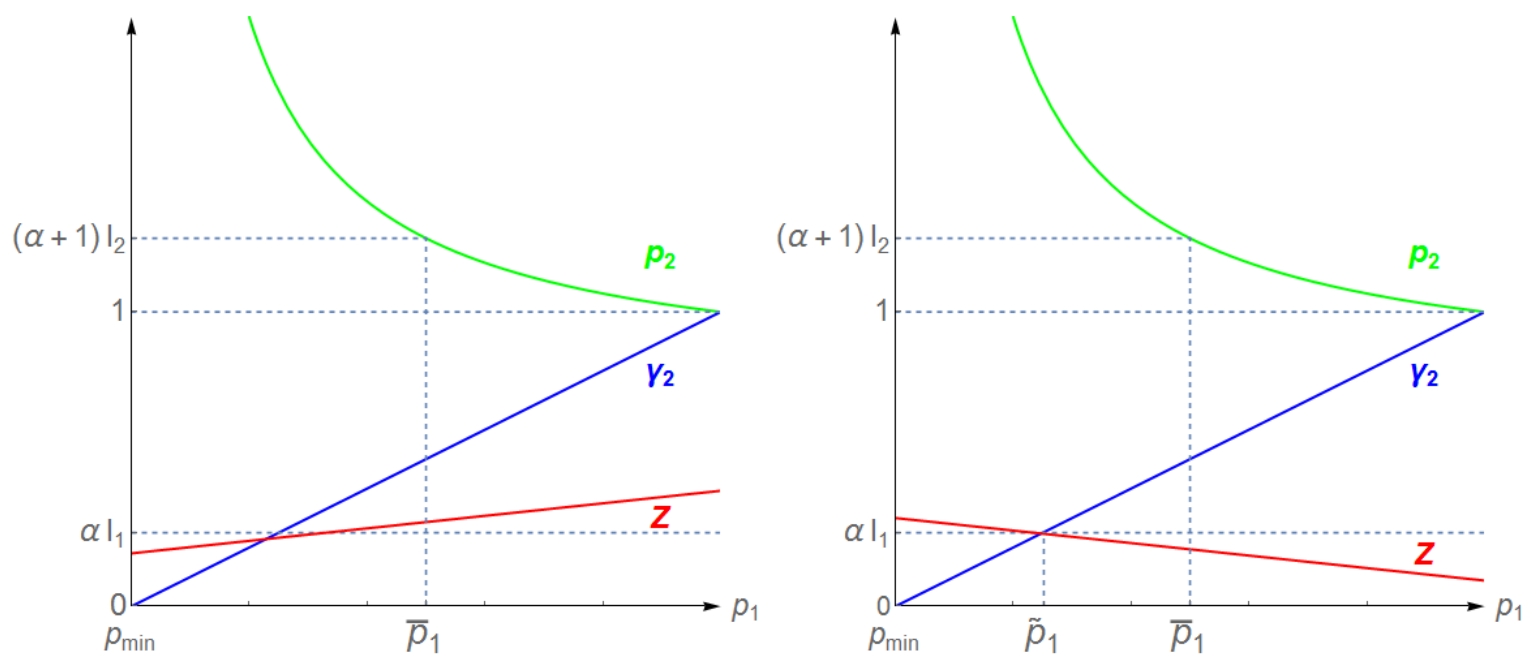

Figure 1: Production by type 2 is feasible if and only if $Z\left(p_{1}\right) \geq \alpha I_{1}$ for some $p_{\min }<$ $p_{1} \leq \bar{p}_{1}$

The conclusions from Proposition 1 now follow from the following observations. Note that $R_{1}$ and $R_{2}$ are strictly increasing in $\alpha$. Given that $I_{1}>0$, straightforward calculations show that $R_{1}(\alpha)=R_{2}(\alpha)$ if and only if $\alpha=\hat{\alpha} \equiv\left(1-I_{2}\right) / I_{1}$, and that $R_{1}(\hat{\alpha})=R_{2}(\hat{\alpha})=\left(1-I_{2}\right) /\left(I_{2}-I_{1}\right) \equiv \bar{R}$. Moreover, $R_{2}-R_{1}$ is strictly increasing at $\alpha=\hat{\alpha}$ :

$$
R_{2}^{\prime}(\hat{\alpha})-R_{2}^{\prime}(\hat{\alpha})=\frac{I_{1} I_{2}\left(1-I_{1}\right)}{\left(I_{2}-I_{1}\right)^{3}}-\frac{I_{1}\left(1-I_{1}\right)}{\left(I_{2}-I_{1}\right)^{2}}=\frac{I_{1}^{2}\left(1-I_{1}\right)}{\left(I_{2}-I_{1}\right)^{3}}>0
$$

Figure 2 illustrates the three different subcases when $I_{2}<1$. Note that the feasible region of $\alpha$ is restricted to $(\underline{\alpha}, \bar{\alpha})$ where $\underline{\alpha}=1 / I_{2}-1$ and $\bar{\alpha}=1 / I_{1}-1$.

\section{References}

Compte, O. and Jehiel, P. (2007). On quitting rights in mechanism design. American Economic Review, 97(2):137-141.

Compte, O. and Jehiel, P. (2009). Veto constraint in mechanism design: Inefficiency with correlated types. American Economic Journal: Microeconomics, 1(1):182-206.

Ellman, M. and Hurkens, S. (2015). Optimal crowdfunding design. SSRN working paper $270961 \%$.

Forges, F. (1999). Ex post individually rational trading mechanisms. In Alkan, A., Aliprantis, C. D., and Yannelis, N. C., editors, Current Trends in Economics: Theory and Applications, pages 157-175. Springer-Verlag, Berlin Heidelberg. 


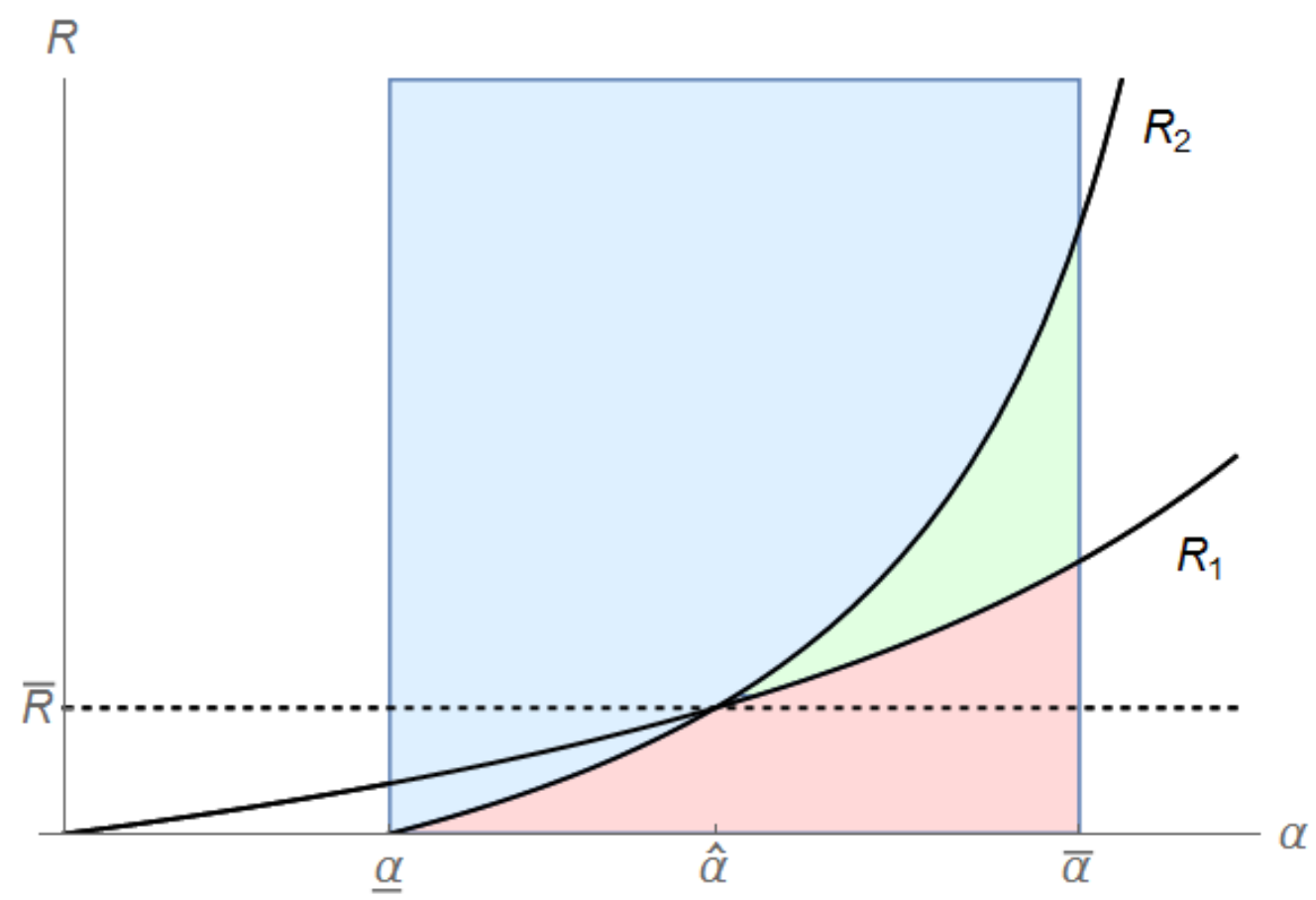

Figure 2: Constrained optima when $I_{2}<1$. In the lower (red) region, there is no production for any type. In the upper green and blue regions, type 1 produces with probability 1 and type 2 with some probability $\gamma_{2} \in(0,1)$. The constrained optima are characterized by the binding constraints $\left(I R^{i}\right),\left(I C_{21}\right)$, and $\left(M H_{2}\right)$ in the large blue region and by $\left(I R^{i}\right),\left(I C_{21}\right)$, and $\left(I C_{12}\right)$ in the smaller green region.

Krähmer, D. and Strausz, R. (2015). Optimal sales contracts with withdrawal rights. The Review of Economic Studies, 82(2):762-790.

Strausz, R. (2017). A theory of crowdfunding: A mechanism design approach with demand uncertainty and moral hazard. American Economic Review, 107(6):1430-76. 